Supporting Information

\title{
Implications from Broadband Microwave Absorption of Metal-modified SiC Fiber Mats
}

Yi Hou, Yong Yang, ${ }^{*}$ Chaoran Deng, Chaojiang Li and Chao-Fu Wang*

Temasek Laboratories, National University of Singapore, 5A Engineering Drive 1, 117411,

Singapore. E-mail: tslyayo@nus.edu.sg (Dr. Y. Yang), cfwang@nus.edu.sg(Dr. Chao-Fu Wang) 


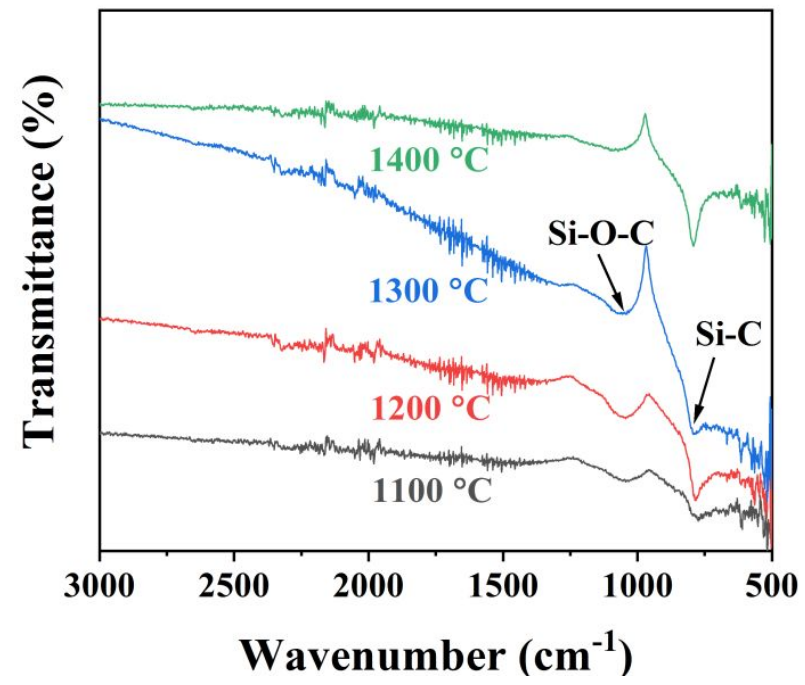

Figure. S1 The FT-IR spectra of $\mathrm{Co} / \mathrm{SiC}$ fiber mats at different pyrolysis temperatures.

The flexibility of fiber mats was characterized by the evolution of resistance changing ratio $(\triangle \mathrm{R} / \mathrm{R} 0)$ under $180^{\circ}$ bending, in which the real-time resistance was monitored by a direct current (DC) resistance tester (Zhi Xin ZX5512, Changzhou, China).
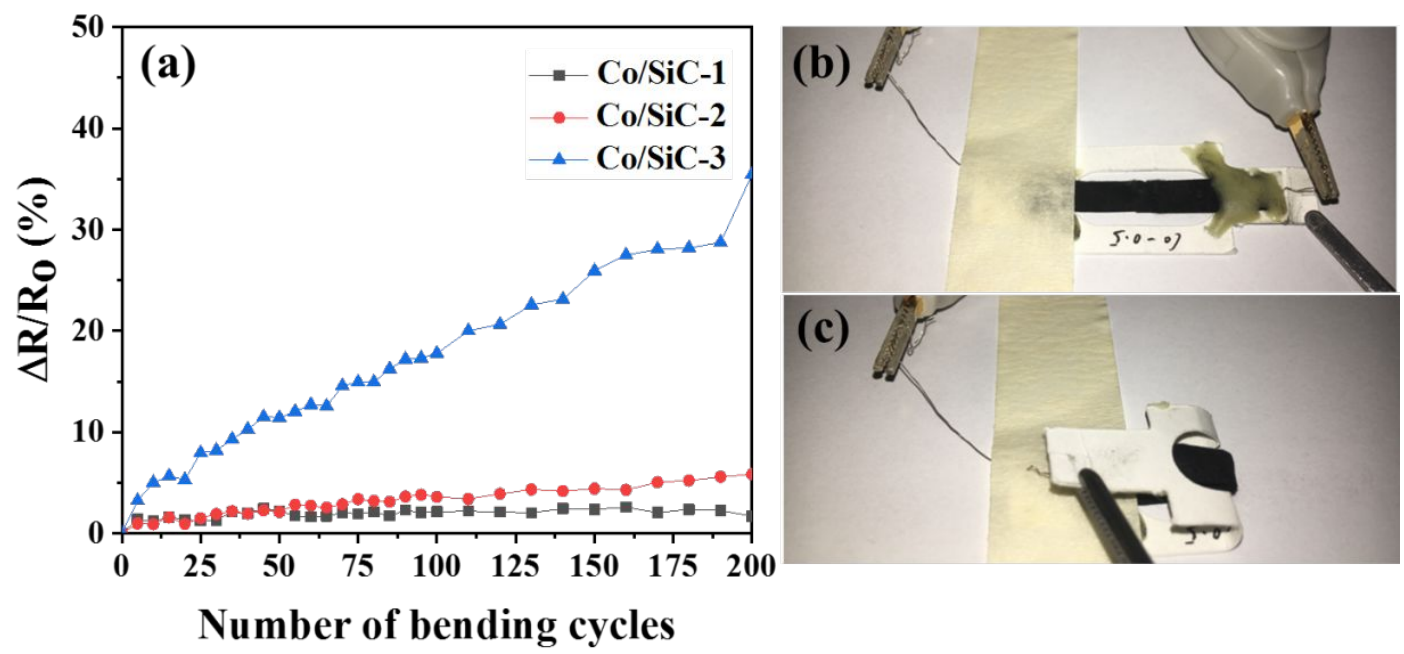

Figure. S2 (a) The changing ratio of electrical resistance $(\Delta R / R)$ under $180^{\circ}$ bending cycles. The inserts are the digital photo of bending testing process: (b) $0^{\circ}$, (c) $180^{\circ}$. 


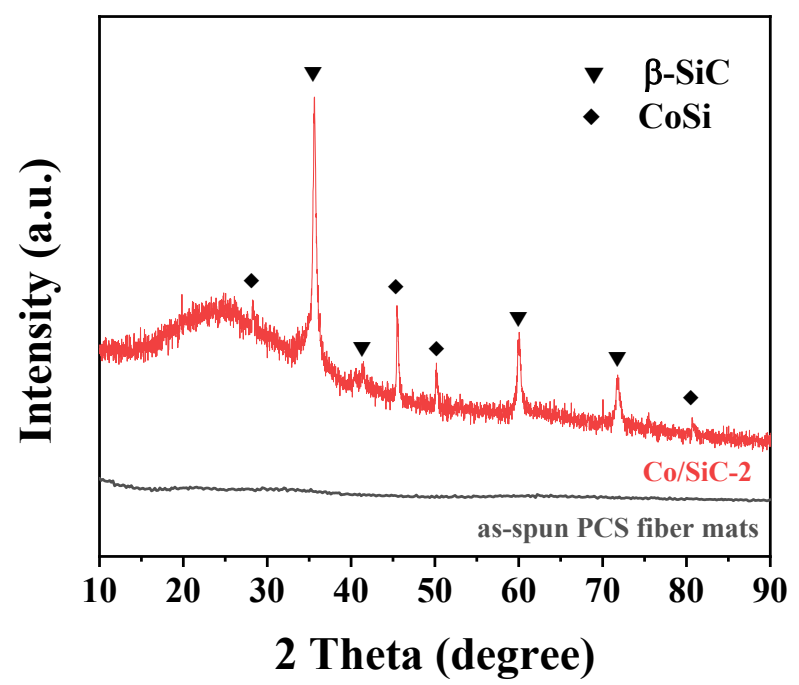

Figure. S3 The XRD patterns of as-spun PCS fiber mats before high temperature pyrolysis. The result of $\mathrm{Co} / \mathrm{SiC}-2$ fiber mats (pyrolyzed at $1400{ }^{\circ} \mathrm{C}$ ) is also provided for comparison.

The as-spun mat shows no diffraction peaks, as compared with the one after high temperature pyrolysis. It indicates that no crystal structure exists in the as-spun mats before pyrolysis.

Table S1 The electrical conductivity of as-spun fiber mats before high temperature pyrolysis. PCS, $\mathrm{Co} / \mathrm{SiC}-1, \mathrm{Co} / \mathrm{PCS}-2$ and $\mathrm{Co} / \mathrm{PCS}-3$ represents the as-spun fiber mats corresponding to pure $\mathrm{SiC}$, $\mathrm{Co} / \mathrm{SiC}-1, \mathrm{Co} / \mathrm{SiC}-2$ and $\mathrm{Co} / \mathrm{SiC}-3$, respectively.

\begin{tabular}{|l|l|l|l|l|}
\hline Sample & PCS & Co/PCS-1 & Co/PCS-2 & Co/PCS-3 \\
\hline Conductivity $(\mathrm{S} / \mathrm{cm})$ & $6.92 \times 10^{-4}$ & $6.91 \times 10^{-4}$ & $6.88 \times 10^{-4}$ & $6.90 \times 10^{-4}$ \\
\hline
\end{tabular}

Though the as-spun mats exhibit higher electrical conductivity than the pyrolyzed SiC fiber mats, it is well below the level of Co modified SiC fiber mats after pyrolysis. 